\title{
EDITORIAL
}

\section{A special collection of reviews on frontiers in immunology}

\author{
Cell Research (2020) 30:827-828; https://doi.org/10.1038/s41422-020-00403-7
}

During this challenging period in human history when SARSCoV-2 has infected more than 20 million people without any sign of an end in sight, we have never been so in need of a healthy immune system to shield ourselves against this highly infectious virus. The reality is that we are still lacking in immunological knowledge to quickly develop therapeutics, either a drug or a vaccine, to slow down the spread of the virus. However, past immunological research has successfully rewarded human society with various immunotherapies, such as the immune checkpoint blockade therapies that have treated numerous cancer patients. The immune system, which intersects with virtually every system in the human body, lies at the center of health and disease. The complexity of the human immune system reflects an ancient Chinese philosophy known as "Yin and Yang". Immune function needs to be robust yet restrained to efficiently clean up external threats without causing selfdamage. When there are "positive" types of immune cells, "negative" types of immune cells must exist simultaneously. At a molecular level, stimulatory and inhibitory molecules reciprocally interplay to elicit well-regulated immune responses. To understand the dynamic and complicated immune responses, immunologists have stepped out of their comfort zones to absorb knowledge from other fields and to apply the newest technologies in their research. Many new branches of immunology have emerged in recent years, including but not limited to, cancer immunology, immunometabolism, microbiota and immunity, neuroimmunology.

Inspired by the fast growth of the relatively "old" immunology field, we are honored to work together with world-renowned immunological experts to present a special review collection in Cell Research discussing our understanding and perspectives of immunological frontiers. Eleven review articles have been assembled to cover diverse topics including cancer immunology, immunometabolism, regulatory $\mathrm{T}$ cells (Treg), innate lymphoid cells (ILCS), microbiota/immune interplay, and the inflammasome.

Cancer immunotherapy can induce a durable response in patients, but the response rates are still low. The primary and secondary resistance is derived either from intrinsic tumor factors or from extrinsic microenvironment factors. Jedd Wolchok at Memorial Sloan Kettering Cancer Center and colleagues provide an overview of existing immunotherapies and discuss two categories of new approaches, i.e. direct (tumor) and indirect (microenvironment) modifiers, to improve the clinical outcome of immunotherapy. ${ }^{1}$ One of the major clinical challenges of immune checkpoint blockade therapies is the development of autoimmune-like immune-related adverse events (irAEs). Vijay Kurchoo at Harvard Medical School and colleagues review the yin and yang of immune checkpoints in cancer and autoimmunity, with an intensive discussion of current approaches and future directions to reduce the risk of irAEs. ${ }^{2}$ In addition to a blockade antibody preventing checkpoint-ligand interaction, other therapeutic reagents inhibiting checkpoint function are under rapid development. Chenqi Xu and a colleague at Shanghai Institute of Biochemistry and Cell Biology review current understandings of checkpoint signaling and discuss alternative paths to target immune checkpoints in cancer. ${ }^{3}$ Innate immune response is an essential arm of antitumor immunity and its roles are less understood than the arm of adaptive immune response. Zhijian Chen at University of Texas Southwestern Medical Center and his colleagues review the antitumor roles of the cGAS-STING pathway and discuss how classic cancer therapies such as radiotherapy and chemotherapy elicit antitumor immune responses through cGAS activation. ${ }^{4}$

Compared with immune signaling, immune metabolism is still at an early developmental stage. However, many other studies have justified the key role of immunometabolism in health and disease. From signaling aspect, immunometabolism is regulated by several serine/threonine kinase-mediated signaling networks, including the PI3K-AGC kinases, mTOR and LKB1-AMPK pathways. Hongbo Chi at St. Jude Children's Hospital and colleagues review these key pathways and also provide perspectives about the pending questions and clinical directions. ${ }^{5}$ Taking on a different angle, Will Bailis at Children's Hospital of Philadelphia, Richard Flavell and colleagues at Yale University synthesize findings regarding the interplay between immune signaling and metabolism. ${ }^{6}$ They discuss "top-down" regulation of metabolic protein expression and function by signal transduction and gene transcription as well as "bottom-up" regulations of the activity of signaling effectors and gene transcription by metabolites. Eva Pålsson-McDermott and Luke O'Neill at Trinity College Dublin discuss targeting immunometabolism as an anti-inflammation strategy. They first review metabolic therapies with anti-inflammatory effects, and then focus on glycolysis and the TCA cycle to prove the concept of targeting metabolic intermediates and enzymes in inflammation. ${ }^{7}$ Besides immunometabolism, inflammasomes serve as key targets for controlling inflammation. Inflammasomes are a group of innate immune receptors sensing invading pathogens and cellular damage to induce production of inflammatory cytokines and pyroptotic cell death. Thirumala-Devi Kanneganti and colleagues at St. Jude Children's Research Hospital review the transcriptional regulation of inflammasome components and related proteins, the post-translational mechanisms of inflammasome activation, and advances in the understanding of the structural basis of inflammasome activation. ${ }^{8}$

In a specific organ, unique populations of immune cells are present to interplay with other tissue-resident cells and environmental factors like microbiota. Formally discovered 10 years ago, ILCs are tissue-resident innate immune cells that resemble transcriptional and functional diversity of the different classes of $T$ cells. Lineage-specifying transcription factors are present to drive certain effector programs. Christoph S. N. Klose at Charité-Universitätsmedizin Berlin and David Artis at Cornell University provide a comprehensive summary and discussion of 
ILC development/heterogeneity and how ILCs impact innate and adaptive immunity. Moreover, influences of the nervous system, circadian rhythm, and developmental plasticity on ILC function have been reviewed. ${ }^{9}$ Eran Elinav at the Weizmann Institute of Science and colleagues extensively review features of microbiome-immunity crosstalk in the intestine and extraintestinal organs. They also highlight the importance of host immune-microbiome interactions in immune-mediated diseases and discuss how to translate current knowledge into future therapeutic interventions. ${ }^{10}$

As a key part of the "yin" arm of the immune system, Treg cells suppress immune response to maintain self-tolerance and homeostasis. Functional abnormality of Treg cells are associated with autoimmunity, cancer and other diseases. Shimon Sakaguchi and a colleague at Osaka University review the transcriptional and epigenetic basis of Treg cell development and function. ${ }^{11}$ In addition to the key transcriptional factor FoxP3, Treg-specific epigenome, such as DNA hypomethylation and histone modifications, is also required to induce stable and functional Treg. This review also summarizes and discusses relevant genetic anomalies or variations in autoimmune diseases as well as therapeutic applications of Treg cells.

In summary, the above review articles highlight the multifaceted organization of the immune system. Local and systematic immune responses provide a natural shield against pathogens and cancers but can also cause diseases if homeostasis is broken. There are more unknowns than knowns for us to explore. These articles are therefore made open access to readers to boost inter-discipline research to unravel the remaining secrets of immunity.
Chenqi Xu $\mathbb{D}^{1,2}$, Hua-Bing $\mathrm{Li}^{3,4}$ and Richard A. Flavell $\mathbb{D}^{5,6}$ ${ }^{1}$ State Key Laboratory of Molecular Biology, CAS Center for Excellence in Molecular Cell Science, Shanghai Institute of Biochemistry and Cell Biology, Chinese Academy of Sciences, Shanghai 200031, China; ${ }^{2}$ School of Life Science and Technology, ShanghaiTech University, Shanghai 200031, China; ${ }^{3}$ Shanghai Institute of Immunology, Department of Liver Surgery, State Key Laboratory of Oncogenes and Related Genes, Ren Ji Hospital, Shanghai Jiao Tong University School of Medicine, Shanghai 200025, China; ${ }^{4}$ Shanghai Jiao Tong University School of Medicine-Yale Institute for Immune Metabolism, Shanghai Jiao Tong University School of Medicine, Shanghai 200025, China; ${ }^{5}$ Department of Immunobiology, Yale School of Medicine, New Haven, CT, USA and ${ }^{6}$ Howard Hughes Medical Institute, New Haven, CT, USA Correspondence: Chenqi Xu (cqxu@sibcb.ac.cn) or Hua-Bing Li (huabing.li@shsmu.edu.cn) or Richard A. Flavell (richard.flavell@yale.edu)

\section{REFERENCES}

1. Murciano-Goroff, Y. R., Warner, A. B. \& Wolchok, J. D. Cell Res. 30, 507-519 (2020).

2. Schnell, A., Bod, L., Madi, A. \& Kuchroo, V. K. Cell Res. 30, 285-299 (2020).

3. He, X. \& Xu, C. Cell Res. 30, 660-669 (2020).

4. Yum, S., Li, M. \& Chen, Z. J. Cell Res. 30, 639-648 (2020).

5. Saravia, J., Raynor, J. L., Chapman, N. M., Lim, S. A. \& Chi, H. Cell Res. 30, 328-342 (2020).

6. Shyer, J. A., Flavell, R. A. \& Bailis, W. Cell Res. 30, 649-659 (2020).

7. Palsson-McDermott, E. M. \& O'Neill, L. A. J. Cell Res. 30, 300-314 (2020).

8. Christgen, S., Place, D. E. \& Kanneganti, T. D. Cell Res. 30, 315-327 (2020).

9. Klose, C. S. N. \& Artis, D. Cell Res. 30, 475-491 (2020).

10. Zheng, D., Liwinski, T. \& Elinav, E. Cell Res. 30, 492-506 (2020).

11. Ohkura, N. \& Sakaguchi, S. Cell Res. 30, 465-474 (2020). 